\title{
Extracting parameters for stellar populations of SDSS galaxy spectra using evolution strategies
}

\author{
Juan C. Gomez and Olac Fuentes \\ Computer Science Department, Instituto Nacional de Astrofísica, Óptica y Electrónica, Luis \\ Enrique Erro 1, Tonantzintla, Puebla 72840, México \\ email: jcgc,fuentes@inaoep.mx
}

Current surveys from modern observatories contain a huge amount of information; in particular, the Sloan Digital Sky Survey (SDSS) has reached the order of terabytes of data in images and spectra. Such amount of information needs to be exploited by sophisticated algorithms that automatically analyze the data in order to extract useful knowledge from the mega databases.

In this work we employ Evolution Strategies (ES), a stochastic optimization method used in other astronomy areas with good results, to automatically extract a set of physical parameters for stellar populations studies (ages, metallicities and reddening) and their relative contributions from a sample of galaxy spectra taken from SDSS. Such parameters are useful in cosmological studies and for understanding galaxy formation and evolution.

Extraction of parameters is treated as an optimization problem and then it is solved by using ES. The idea is to reconstruct each galaxy spectrum from the sample by means of a linear combination of three simple theoretical models of stellar population synthesis (young, intermediate and old). The goal of the fitting task with ES is to find, efficiently and automatically, the best combination for the redenning $\left(r_{1}, r_{2}, r_{3}\right)$, metallicities $\left(m_{1}, m_{2}, m_{3}\right)$ ages $\left(a_{1}, a_{2}, a_{3}\right)$ and relative contributions $\left(c_{1}, c_{2}, c_{3}\right)$ to produce a model that matches the original spectrum. Model is contructed using the equation: $\mathbf{g}_{\lambda}=\sum_{i=1}^{3} c_{i} s_{i}\left(a_{i}, m_{i}, \lambda\right)\left(10^{-4 r_{i} k}\right)$, where $s_{i}\left(a_{i}, m_{i}, \lambda\right)$ represents a model for specific age and specific metallicity and $10^{-4 r_{i} k}$ is the redenning term.

The process starts with an observed spectrum $\mathbf{o}_{\lambda}$ that is passed to a preprocessing module to be adjust by rest frame, redenning correction, cut, re-bin and normalization; afterwards we begin an iterative process, which starts with a set of randomly created population synthesis parameters that is passed to a model creator program. This module produce a modeled spectrum $\left(\mathbf{g}_{\lambda}\right)$ that is compared with the original spectrum. If we obtain a good fit we stop the process otherwise we modify the parameters using ES operators (mutation an cross-over) and we return to create another model.

Our implementation uses a set of 50 models taken from Bressan et al. (1994) while all the spectra used were cut in order to cover the $3800 \AA$ - $8000 \AA$ range. Bressan's models cover the following ages: young $=\left[10^{6}, 10^{6.3}, 10^{6.6}, 10^{7}, 10^{7.3}\right] \mathrm{yr}$, intermediate $=\left[10^{7.6}, 10^{8}\right.$, $\left.10^{8.3}\right] \mathrm{yr}$, old $=\left[10^{9}, 10^{10.2}\right] \mathrm{yr}$ and the following metallicities: $[0.0004,0.004,0.008,0.02$, $0.05] \mathrm{Z}_{\odot} \cdot$

Until now we have obtained results for a set of 50 spectra from SDSS Data Release 2 as a first approach to the use of this method, proving ES are very well suited to extract stellar population parameters from galaxy spectra. 